\title{
Proximal shift of colorectal cancer with increasing age in different ethnicities
}

This article was published in the following Dove Press journal:

Cancer Management and Research

\author{
Lin Yang ${ }^{1-3}$ \\ Zhenchong Xiong ${ }^{1-3}$ \\ Wenzhuo $\mathrm{He}^{1-3}$ \\ Kunqian $\mathrm{Xie}^{1-3}$ \\ Shousheng Liul-3 \\ Pengfei Kong ${ }^{1-3}$ \\ Chang Jiang ${ }^{1-3}$ \\ Guifang Guo ${ }^{1-3, *}$ \\ Liangping $\mathrm{Xia}^{1-3, *}$ \\ 'Department of VIP, Sun Yat-sen \\ University Cancer Center, Guangzhou, \\ China; ${ }^{2}$ State Key Laboratory of \\ Oncology in Southern China, \\ Guangzhou, China; ${ }^{3}$ Collaborative \\ Innovation Center for Cancer \\ Medicine, Guangzhou, China \\ *These authors contributed equally to \\ this work
}

Correspondence: Guifang Guo

Sun Yat-sen University Cancer Center,

65I Dongfeng Road East, Guangzhou

510060, China

Email guogf@sysucc.org.cn

Liangping Xia

Sun Yat-sen University Cancer Center,

65 I Dongfeng Road East, Guangzhou

510060, China

Email xialp@sysucc.org.cn
Background: Studies have indicated a variation in colon cancer pathology with increased age. More findings have also suggested differences in genetics, biology, and demography in terms of ethnicity. Large-scale studies closely examining tumor location shift with aging and ethnicity are scarce.

Objective: We compared the tumor location shift with aging and the difference in survival based on tumor location by age group among the African-American, White, and Asian/Pacific Islander patients with colorectal cancer.

Materials and methods: We collected 270,390 cases from the Surveillance, Epidemiology, and End Results database between 2004 and 2014. Ethnicity distribution between younger (age $<70$ years) and older (age $\geq 70$ years) patients was analyzed using univariate and multivariate logistic regression. The Kaplan-Meier method was used to compare the tumor location survival difference in the African-American, White, and Asian/Pacific Islander patients.

Results: Larger tumors, female sex, M0, advanced N stage, no treatment, moderate to poor differentiation, total number of lymph nodes evaluated $>12$, and right-sided colon cancer were more common in patients aged $\geq 70$ years. More adverse prognosis was found in younger patients compared to older patients. Tumor location frequency differed based on age; the most pronounced differences were found in White patients. The right-sided colon cancer survival inferiority was present only in White patients.

Conclusion: Our findings support the premise of etiological and carcinogenic differences based on tumor location and between younger and older patients.

Keywords: age, proximal shift, tumor location, ethnicity, SEER, colorectal cancer

\section{Introduction}

Colorectal cancer (CRC) contributes greatly to cancer mortality and morbidity worldwide. ${ }^{1}$ Diverse morphologic features and behavior have been reported for this disease. Many studies have suggested a difference in terms of genetics, biology, and demography between right and left-sided CRC (RCC and LCC, respectively). RCC and LCC are viewed as two different lesions and their biological and demographic characteristics differ. ${ }^{2}$

Ethnicity is an important contributing factor among the variables affecting the incidence rates of $\mathrm{CRC}$. There are racial disparities in the CRC incidence among African-Americans and Hispanics. ${ }^{3}$ African-Americans form a unique cohort of patients for whom CRC data, trends, and characteristics remain unclear. ${ }^{4}$ African-Americans are more likely to be diagnosed with advanced stage CRC and to have poorer survival 
rates after diagnosis compared to Whites. ${ }^{5}$ On the basis of the results of clinical trials in the USA with a high proportion of non-Hispanic Whites, various adjuvant chemotherapy regimens for colon cancer have been introduced in East Asian countries. ${ }^{6}$ Hispanics have a lower overall incidence rate of CRC as compared to non-Hispanic Whites. ${ }^{4}$ The survival rates of African-American patients diagnosed with colon cancer is considerably lower than that of White patients. ${ }^{7}$ To better understand the results of numerous studies and clinical trials performed worldwide, it would be useful to determine potential prognostic differences due to race/ethnicity after surgery for colon cancer.

Many factors contribute to CRC incidence, including ethnicity, sex, diet/environment, and socioeconomic status. Many of these variables have been explored, but many unanswered questions remain. The $\mathrm{CRC}$ incidence rate increases with age. ${ }^{8,9}$ However, information on the $\mathrm{RCC} / \mathrm{LCC}$ ratio disparities among different ethnicities is rare. Understanding the age-related changes in the clinicopathologic features of $\mathrm{CRC}$ is very important for the implementation of age-related preventive/therapeutic measures. The study may also give a clue that the patients' inclusion in clinical trials should consider not only tumor location but also ethnicity, given the different reactions of patients of different ethnicities to targeted agents since there is a difference in race in the population that benefited from the bevacizumab trial. ${ }^{10-12}$

Although several reports have documented the characteristics of CRC in the elderly, to the best of our knowledge, ethnicity-specific, age-related changes in CRC characteristics, especially those focusing on detailed cancer localization and ethnicity, have not been fully investigated. The present study aimed to clarify the ethnicity-specific, age-related changes in the clinicopathologic features of CRC.

\section{Materials and methods \\ Samples}

The study was conducted based on the Surveillance, Epidemiology, and End Results (SEER) programs and did not require review board approval. The SEER registries (Connecticut, Iowa, Hawaii, New Mexico, Utah, and the metropolitan areas of Atlanta, Detroit, San Francisco-Oakland, and Seattle Puget Sound) represent $\sim 9 \%$ of the US population. ${ }^{13}$ We collected the information of individuals with pathologically diagnosed disease from 2004 to June 2012. All patients had a first diagnosis of invasive, non-distant, metastatic colon cancer according to the American Joint Committee on Cancer sixth edition criteria. All patients had pathologically confirmed adenocarcinoma or mucinous adenocarcinoma, and tumors were graded based on definition, that is, as well, moderate, poor, or undifferentiated. Colon cancer was evaluated as right sided (transverse colon, hepatic flexure, ascending colon, cecum), left sided (sigmoid colon, descending colon, splenic flexure), or rectosigmoid colon (rectum, rectosigmoid junction). The total number of lymph nodes (TNLN) evaluated was recorded and they were considered categorical variables if the node number was $=12$. The SEER nine dataset categorized ethnicity as White, African-American, Native American/Alaska Native, Asian/Pacific Islander, and unknown. ${ }^{14}$ As the sample for Native American/Alaska Native patients was small, ${ }^{14-16}$ the data for this group were not analyzed.

Patients were observed from 6 months after the first diagnosis of colon cancer until the last follow-up, death, or the end of the study, whichever occurred first.

\section{Statistical analysis}

Univariate logistic analysis was used to assess the correlation of age and clinicopathologic variables, tumor depth (Tis-T2, T3-T4), histologic type (well/moderate/poor differentiation), regional lymph node metastasis, distant metastasis, ethnicity, and tumor size. The correlation between patient age and clinicopathologic characteristics was evaluated by parametric and nonparametric methods. The parametric method was carried out for variables of sex, tumor depth, distant metastasis, chemotherapy, and pathologic type using the unpaired Student's $t$-test. The nonparametric method was used for the ordered variables such as regional lymph node metastasis, radiotherapy, pathology grade, the number of lymph nodes retrieved, tumor size, and the tumor location using the Spearman's rank correlation coefficient. Significant variables affecting patient age were assessed by multivariate analysis using a logistic regression model. Kaplan-Meier survival analysis was used to compare the difference in survival based on tumor location. All analyses were performed with SPSS version 13.0, and a two-sided value of $P<0.05$ was considered statistically significant.

\section{Ethics approval}

This article does not contain any studies with human participants or animals performed by any of the authors.

\section{Results}

\section{Patients' characteristics}

Overall, there were 270,390 patients in our cohort. Table 1 lists the general patient features. The patient age ranged 11-85 years (mean age, 68 \pm 13.1 years); 30,905 patients (11.4\%) were African-Americans, 218,495 (80.8\%) were Whites, and the remaining 20,990 (7.8\%) were Asian/Pacific 
Table I Univariate analysis of the association between clinicopathologic variables and age in patients of different races

\begin{tabular}{|c|c|c|c|c|c|c|c|c|c|}
\hline \multirow[t]{2}{*}{ Patient characteristics } & \multicolumn{3}{|c|}{ African-American } & \multicolumn{3}{|l|}{ White } & \multicolumn{3}{|c|}{ Asian/Pacific Islander } \\
\hline & $\mathbf{n}$ & $\begin{array}{l}\text { Mean age } \\
\text { (years) }\end{array}$ & $P$-value & $\mathbf{n}$ & $\begin{array}{l}\text { Mean age } \\
\text { (years) }\end{array}$ & $P$-value & $\mathbf{n}$ & $\begin{array}{l}\text { Mean age } \\
\text { (years) }\end{array}$ & $P$-value \\
\hline \multirow[t]{2}{*}{ Sex } & & & $<0.001$ & & & $<0.001$ & & & $<0.001$ \\
\hline & & & $<0.001$ & & & & & & \\
\hline Male & 15,078 & $63 \pm 12$ & & 112,370 & $68 \pm 13$ & & 10,706 & $65 \pm 13$ & \\
\hline Female & 15,827 & $65 \pm 13$ & & 106,125 & $71 \pm 13$ & & 10,284 & $67 \pm 14$ & \\
\hline T stage & & & 0.989 & & & $<0.001$ & & & 0.974 \\
\hline Tis, T0, TI, T2 & 8,842 & $65 \pm 12$ & & 66,115 & $69 \pm 12$ & & 6.068 & $66 \pm 12$ & \\
\hline T3-T4 $(4,5)$ & 22,063 & $64 \pm 13$ & & 152,380 & $69 \pm 13$ & & 14,922 & $66 \pm 14$ & \\
\hline N stage & & & $<0.001$ & & & $<0.001$ & & & $<0.001$ \\
\hline No & 16,873 & $65 \pm 12$ & & 127,545 & $7 I \pm 13$ & & 11,254 & $67 \pm 13$ & \\
\hline $\mathrm{NI}$ & 8,648 & $64 \pm 13$ & & 54,227 & $68 \pm 13$ & & 5,805 & $65 \pm 13$ & \\
\hline N2 & 5,384 & $62 \pm 12$ & & 36,723 & $66 \pm 14$ & & 3,931 & $63 \pm 14$ & \\
\hline Distant metastasis & & & $<0.001$ & & & $<0.001$ & & & $<0.001$ \\
\hline Mo & 25,865 & $65 \pm 13$ & & 190,352 & $70 \pm 13$ & & 18,208 & $66 \pm 13$ & \\
\hline MI & 5,040 & $62 \pm 13$ & & 28,143 & $65 \pm 14$ & & 2,782 & $63 \pm 14$ & \\
\hline Chemotherapy & & & $<0.001$ & & & $<0.001$ & & & $<0.001$ \\
\hline No & 19,025 & $67 \pm 12$ & & 139,239 & $74 \pm 12$ & & $|2,26|$ & $70 \pm 13$ & \\
\hline Yes & 11,880 & $59 \pm 12$ & & 79,256 & $62 \pm 13$ & & 8,729 & $60 \pm 13$ & \\
\hline Radiotherapy & & & $<0.001$ & & & $<0.001$ & & & $<0.001$ \\
\hline No & 64 & $67 \pm 13$ & & 623 & $74 \pm 13$ & & 66 & $70 \pm 14$ & \\
\hline Yes & 2,907 & $59 \pm 12$ & & 26,112 & $61 \pm 12$ & & 2,959 & $59 \pm 13$ & \\
\hline Unknown & 27,934 & $65 \pm 13$ & & 191,760 & $71 \pm 13$ & & 17,965 & $67 \pm 13$ & \\
\hline Pathology grade & & & $<0.001$ & & & $<0.001$ & & & $<0.001$ \\
\hline Well differentiated & 2,735 & $65 \pm 12$ & & 18,756 & $69 \pm 13$ & & $\mathrm{I}, 475$ & $65 \pm 13$ & \\
\hline Moderately differentiated & 21,740 & $64 \pm 13$ & & 146,085 & $69 \pm 13$ & & $|4,93|$ & $66 \pm 13$ & \\
\hline Poorly differentiated & 4,364 & $64 \pm 13$ & & 38,025 & $7 I \pm 14$ & & 3,392 & $65 \pm 15$ & \\
\hline Undifferentiated & 539 & $63 \pm 14$ & & 5,434 & $7 I \pm 14$ & & 309 & $65 \pm 15$ & \\
\hline Unknown & $\mathrm{I}, 527$ & $63 \pm 12$ & & 10,195 & $66 \pm 13$ & & 882 & $63 \pm 13$ & \\
\hline Pathologic type & & & 0.010 & & & $<0.001$ & & & $<0.001$ \\
\hline Adenocarcinoma & 30,522 & $64 \pm 12$ & & 215,169 & $69 \pm 12$ & & 20,774 & $66 \pm 13$ & \\
\hline Mucinous carcinoma & 383 & $63 \pm 13$ & & 3,326 & $68 \pm 13$ & & 216 & $67 \pm 14$ & \\
\hline Number of lymph nodes & & & $<0.001$ & & & $<0.001$ & & & $<0.001$ \\
\hline$<12$ & 8,618 & $66 \pm 12$ & & 72,386 & $70 \pm 12$ & & 7,235 & $68 \pm 13$ & \\
\hline$\geq 12$ & 22,287 & $64 \pm 13$ & & 146,109 & $69 \pm 13$ & & 13,755 & $65 \pm 14$ & \\
\hline Tumor site & & & $<0.001$ & & & $<0.001$ & & & $<0.001$ \\
\hline $\mathrm{RCC}$ & 16,413 & $66 \pm 12$ & & 107,706 & $73 \pm 12$ & & 7,572 & $70 \pm 13$ & \\
\hline LCC & 9,129 & $63 \pm 12$ & & 60,004 & $67 \pm 13$ & & 7,635 & $65 \pm 13$ & \\
\hline Rectum & 5,363 & $61 \pm 12$ & & 50,785 & $64 \pm 13$ & & 5,783 & $62 \pm 13$ & \\
\hline Tumor sizes (mm) & & & $<0.001$ & & & $<0.001$ & & & $<0.001$ \\
\hline$\leq 40$ & 12,958 & $65 \pm 12$ & & 97,720 & $70 \pm 13$ & & 10.009 & $67 \pm 13$ & \\
\hline $40-70$ & 11,227 & $64 \pm 13$ & & 73,224 & $70 \pm 14$ & & 6,941 & $66 \pm 14$ & \\
\hline$\geq 70$ & 6,720 & $63 \pm 13$ & & 47,551 & $67 \pm 14$ & & 4,040 & $64 \pm 14$ & \\
\hline
\end{tabular}

Abbreviations: LCC, left-sided colon cancer; RCC, right-sided colon cancer.

Islanders. Figure 1 shows the ethnicity variation according to age. The sample size variation in this study could be accepted according to the study by Siegel et al. ${ }^{7}$ Additionally, we compared all the variants among the ethnic groups (Table S1).

\section{Univariate analysis and multivariate analysis}

Table 1 shows the results of the univariate analysis of the association between the clinicopathologic variables and age, according to ethnicity, in patients with CRC. The AfricanAmerican patients were older in cases with $\mathrm{RCC}(P<0.001)$, of female sex $(P<0.001)$, with advanced $\mathrm{N}$ stage $(P<0.001)$, without M1 $(P<0.001)$, without treatment $(P<0.001)$, and with poor differentiation $(P<0.001)$, TNLN $<12(P<0.001)$, and smaller tumor size $(P<0.001)$. No correlation between patient age and the other clinicopathologic variables was observed. In Asian/ Pacific Islander and White patients, the age-associated variables were similar to those of the African-American patients. 
Race disparitles in different age

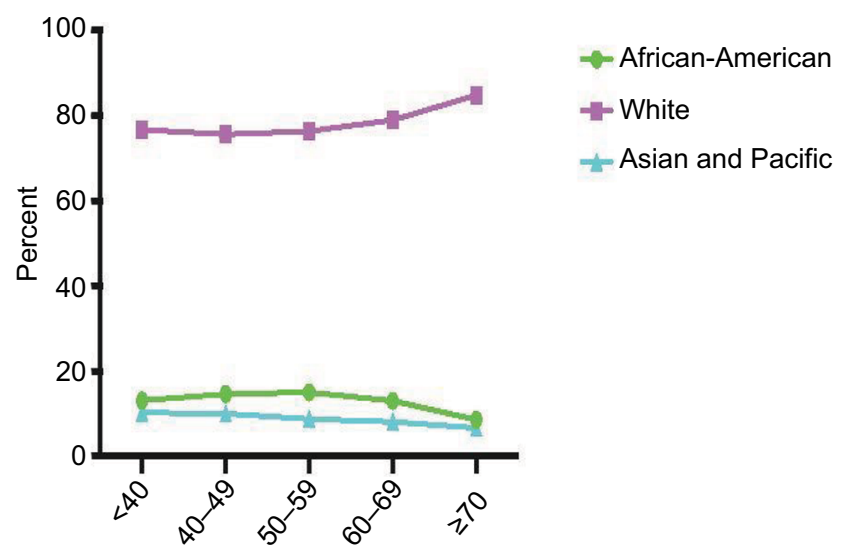

Figure I African-American, White, and Asian/Pacific Islander patients in each age group.

Note: The White ethnicity ratio increased with patient age, and this tendency was more prominent in the population aged $\geq 70$ years.
Independent variables correlating with age $\geq 70$ years were identified using multivariate logistic regression analysis (Table 2). In the African-American patients, female sex (odds ratio $[\mathrm{OR}]=1.28, P<0.001$ ), late $\mathrm{N}$ stage (N1 vs No, $\mathrm{OR}=0.424)$; $\mathrm{M} 0$ stage $(\mathrm{OR}=0.804, P<0.001)$, without chemotherapy $(\mathrm{OR}=0.304, P<0.001)$, pathology grade (moderate vs well, OR=1.120, $P=0.010$; poor vs well, $\mathrm{OR}=1.266, P<0.001$; undifferentiated vs well, $\mathrm{OR}=1.352$, $P=0.004)$, TNLN $<12(\mathrm{OR}=0.712, P<0.001)$, and $\mathrm{RCC}$ (LCC vs RCC, OR=0.672, $P<0.001$; rectal cancer vs RCC, OR=0.696, $P<0.001)$ were independent variables correlated with higher age. Similarly, in White and Asian/ Pacific Islander patients, the presence of advanced $\mathrm{T}$ stage and smaller tumor size were additional independent variables.

Table 2 Multivariate logistic regression analysis for identifying independent variables correlating with patient age $\geq 70$ years

\begin{tabular}{|c|c|c|c|c|c|c|}
\hline \multirow[t]{2}{*}{ Patient characteristic } & \multicolumn{2}{|l|}{ African-American } & \multicolumn{2}{|l|}{ White } & \multicolumn{2}{|c|}{ Asian/Pacific Islander } \\
\hline & OR (95\% Cl) & $P$-value & OR (95\% Cl) & $P$-value & OR (95\% CI) & $P$-value \\
\hline \multicolumn{7}{|l|}{ Sex } \\
\hline Female vs male & $1.275(1.215-1.339)$ & $<0.001$ & $1.332(1.308-1.357)$ & $<0.001$ & I.165 (1.099-1.235) & $<0.001$ \\
\hline \multicolumn{7}{|l|}{ T stage } \\
\hline T3-T4 vs Tis, T0-T2 & & & 1.514 (I.480-I.549) & $<0.00$ I & 1.375 (1.278-1.480) & $<0.001$ \\
\hline N stage & & $<0.001$ & & $<0.00$ I & & $<0.001$ \\
\hline No & 1.000 & & 1.000 & & 1.000 & \\
\hline $\mathrm{NI}$ & $1.424(1.337-1.517)$ & $<0.001$ & $1.259(1.228-1.29 \mid)$ & $<0.001$ & I.364 (I.26I-I.475) & $<0.001$ \\
\hline N2 & $1.264(1.168-1.368)$ & $<0.001$ & I.I05 (1.072-I.139) & $<0.00$ I & I.165 (I.06I-I.280) & 0.001 \\
\hline \multicolumn{7}{|l|}{ Distant metastasis } \\
\hline Yes vs no & $0.804(0.746-0.867)$ & $<0.001$ & $0.769(0.746-0.793$ & $<0.001$ & $0.783(0.7 \mid I-0.862)$ & $<0.001$ \\
\hline \multicolumn{7}{|l|}{ Chemotherapy } \\
\hline Yes vs no & $0.304(0.286-0.323)$ & $<0.001$ & $0.250(0.244-0.256)$ & $<0.001$ & $0.284(0.265-0.305)$ & $<0.001$ \\
\hline Radiotherapy & & & & $<0.001$ & & \\
\hline No & & & 1.000 & & & \\
\hline Yes & & & $0.672(0.565-0.799)$ & $<0.001$ & & \\
\hline Unknown & & & $0.725(0.6 \mathrm{II}-0.860)$ & $<0.001$ & & \\
\hline Pathology grade & & & & $<0.001$ & & $<0.001$ \\
\hline Well differentiated & 1.000 & & 1.000 & & 1.000 & \\
\hline Moderately differentiated & $1.120(1.027-1.221)$ & 0.010 & $1.139(1.102-1.177)$ & $<0.001$ & $1.309(1.165-1.470)$ & $<0.001$ \\
\hline Poorly differentiated & $1.266(1.139-1.407)$ & $<0.001$ & $1.386(1.333-1.44 I)$ & $<0.001$ & $1.308(1.142-1.499)$ & $<0.001$ \\
\hline Undifferentiated & $1.352(1.104-1.655)$ & 0.004 & $1.426(1.335-1.524)$ & $<0.001$ & $1.430(1.094-1.869)$ & 0.009 \\
\hline Unknown & $0.85 I(0.740-0.979)$ & 0.024 & $0.902(0.856-0.95 I)$ & $<0.001$ & $1.037(0.862-0.249)$ & 0.699 \\
\hline \multicolumn{7}{|l|}{ Pathologic type } \\
\hline Mucinous carcinoma vs adenocarcinoma & $0.699(0.556-0.879)$ & 0.002 & $0.695(0.645-0.750)$ & $<0.001$ & & \\
\hline Number of lymph nodes & & & & & & $<0.001$ \\
\hline$\geq 12$ vs $<12$ & $0.712(0.674-0.752)$ & $<0.001$ & $0.7 \mathrm{II}(0.697-0.725)$ & $<0.001$ & $0.656(0.616-0.699)$ & $<0.001$ \\
\hline Tumor size (mm) & & $<0.001$ & & $<0.001$ & & $<0.001$ \\
\hline$\leq 40$ & 1.000 & & 1.000 & & 1.000 & \\
\hline $40-70$ & $1.076(1.018-1.138)$ & 0.010 & $1.002(0.980-1.024)$ & 0.868 & $0.963(0.899-1.033)$ & 0.292 \\
\hline$\geq 70$ & $0.845(0.79 \mid-0.902)$ & $<0.001$ & $0.819(0.799-0.839)$ & $<0.001$ & $0.775(0.7 \mid 4-0.840)$ & $<0.001$ \\
\hline Tumor site & & $<0.001$ & & $<0.001$ & & $<0.001$ \\
\hline $\mathrm{RCC}$ & 1.000 & & 1.000 & & 1.000 & \\
\hline LCC & $0.672(0.635-0.7 \mathrm{II})$ & $<0.001$ & $0.507(0.496-0.518)$ & $<0.001$ & $0.536(0.500-0.574)$ & $<0.001$ \\
\hline Rectum & $0.696(0.647-0.748)$ & $<0.001$ & $0.502(0.489-0.516)$ & $<0.001$ & $0.537(0.497-0.58 I)$ & $<0.001$ \\
\hline
\end{tabular}

Abbreviations: LCC, left-sided colon cancer; OR, odds ratio; RCC, right-sided colon cancer. 
RCC, female sex, TNLN $<12$, no to moderate differentiation, late $\mathrm{N}$ stage, and M0 stage without chemotherapy were the common independent variables with age $\geq 70$ years in the African-American, White, and Asian/Pacific Islander patients.

\section{Variation of RCC ratio with age}

The $\mathrm{RCC} /$ rectum or $\mathrm{RCC} / \mathrm{LCC}$ ratio was relatively low in patients aged $<70$ years, but was increased in patients aged $\geq 70$ years (Table 3 ). Tumor location was an independent factor associated with patient age in all three ethnic groups. Figure 2 shows the distribution of RCC tumors according to the age group. The shift of preferential tumor location to the proximal colon with increased age was more prominent in White patients than in African-American and Asian/ Pacific Islander patients. In White patients, only $27.2 \%$ and $23.4 \%$ of patients aged $<60$ and $60-69$ years, respectively,

Table 3 Location of new CRC according to age group

\begin{tabular}{lllllll}
\hline $\begin{array}{l}\text { Age } \\
\text { (years) }\end{array}$ & Total & Right & Left & Rectum & $\begin{array}{l}\text { Right/ } \\
\text { left }\end{array}$ & $\begin{array}{l}\text { Right/ } \\
\text { rectum }\end{array}$ \\
\hline$<40$ & 8,087 & 2,688 & 2,680 & 2,719 & $1.00^{*}$ & $0.99 *$ \\
$40-49$ & 19,787 & 6,313 & 6,624 & 6,850 & $0.95^{*}$ & $0.92^{*}$ \\
$50-59$ & 50,231 & 18,151 & 16,706 & 15,374 & $1.09^{*}$ & $1.18^{*}$ \\
$60-69$ & 64,942 & 29,346 & 19,419 & 16,177 & $1.5 I^{*}$ & $1.8 I^{*}$ \\
$\geq 70$ & 127,343 & 75,193 & 31,339 & 20,811 & $2.40^{*}$ & $3.61^{*}$ \\
Total & 270,390 & 131,691 & 76,768 & 61,931 & $1.72^{*}$ & $2.13^{*}$ \\
\hline
\end{tabular}

Notes: *The proportion of right-sided lesions increased with age $(P=0.01$, by chisquared trend test). Left column reflects left-sided colon cancer; right column reflects right-sided colon cancer. The Right/left column reflects the means the ratio of right-sided colon cancer to left-sided and the Right/rectum column reflects the ratio of right-sided colon cancer to rectum cancer.

Abbreviation: CRC, colorectal cancer.

Whole cohort

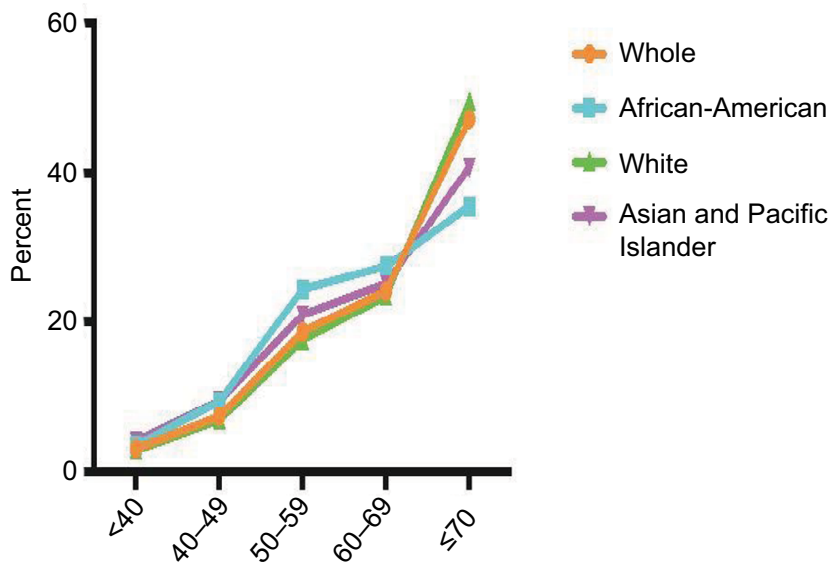

Figure 2 Distribution of tumor location according to age group in the whole cohort and in the African-American, White, and Asian/Pacific Islander patients, respectively. Notes: The shift for the proximal predominance of CRC with patient age was more remarkable in White patients than in African-American and Asian/Pacific Islander patients.

Abbreviation: $\mathrm{CRC}$, colorectal cancer. had RCC, but this increased to $49.4 \%$ in patients aged $\geq 70$ years. In Asian/Pacific Islander patients, only $34.3 \%$ and $25 \%$ of patients aged $<60$ and $60-69$ years, respectively, had RCC, but this increased to $40.7 \%$ in patients aged $\geq 70$ years. The correlation between tumor location and age was stronger in White and Asian/Pacific Islander patients than in African-American patients $(P<0.001)$. In African-American patients, $28.9 \%$ and $24 \%$ of patients aged $<60$ and $60-69$ years, respectively, had RCC, and it increased to $35.5 \%$ in the patients aged $\geq 70$ years

The RCC shift with age was present in the subgroup (tumor stage, differentiation, tumor size, TNLN, sex) analysis (Figures S1-3 for African-Americans, Asian/Pacific patients and Whites, respectively). We noted a big shift in RCC in White patients aged $\geq 70$ years, and the significance remained in the subgroup analysis. The RCC ratio was increased at least two times in the patients aged $\geq 70$ years compared with the patients aged $<70$ years.

\section{Survival comparison}

In the African-American patients, there was no survival difference between RCC, LCC, and rectal cancer in each age group (Figure 3A). In the Asian/Pacific Islander patients, RCC survival was relatively poorer only in the 50-59 years age group (Figure 3B). However, in White patients, RCC survival was poor in the age groups 40-49, 50-59, 60-69, and $\geq 70$ years (Figure $3 \mathrm{C}$ ). RCC survival difference inferiority was most apparent in the Whites.

\section{Discussion}

In our study, RCC was an independent variable correlated with age $\geq 70$ years in the African-American, White, and Asian/Pacific Islander patients with CRC. The significance remained in the subgroup analysis, especially in White patients. Additionally, the RCC survival disadvantage as compared to $\mathrm{LCC} /$ rectal cancer was present only in the White patients.

Early-stage tumors and low-grade phenotype are more frequent in older patients. ${ }^{17-19}$ The present study reveals a higher percentage of poorly differentiated, lymph nodepositive, and non-M1 tumors in patients aged $\geq 70$ years, which is consistent with previous studies. Although we are uncertain why these discrepancies exist, both environmental and genetic factors might play a role in it.

The detection of colon cancer is rare in people aged $<50$ years, which is similar to previous reports. ${ }^{17,18}$ Here, we observed that the RCC ratio increased gradually with age, which agrees with previous population-based reports. ${ }^{2}$ 
A

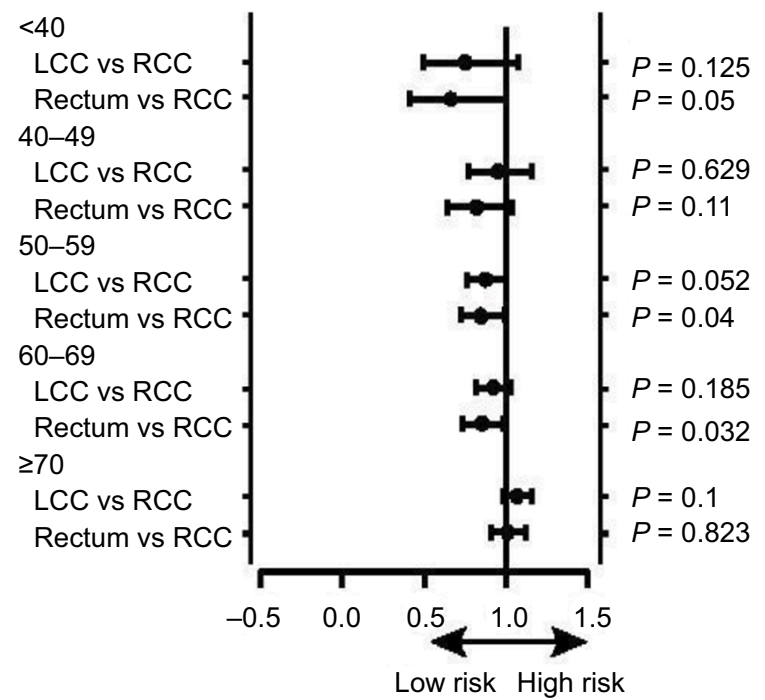

C

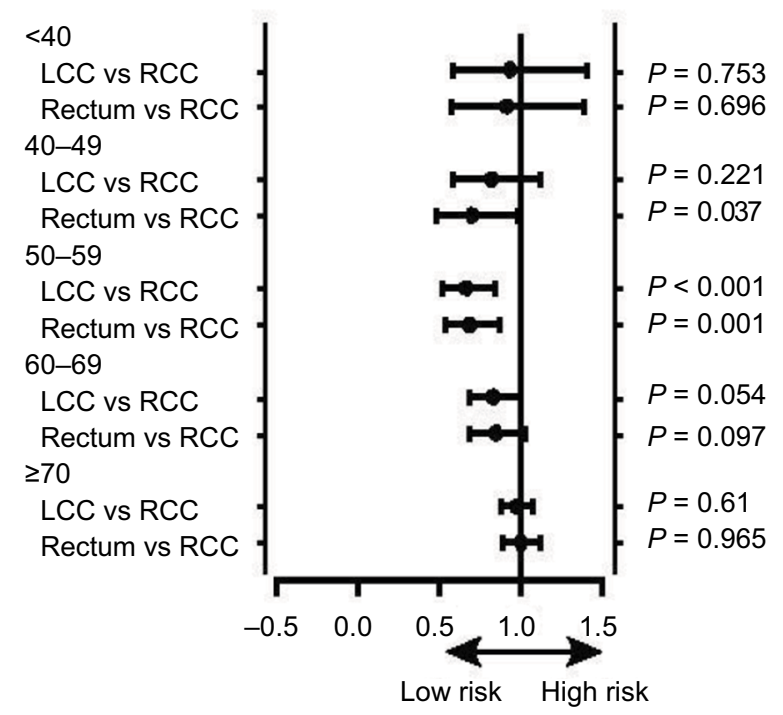

B

White

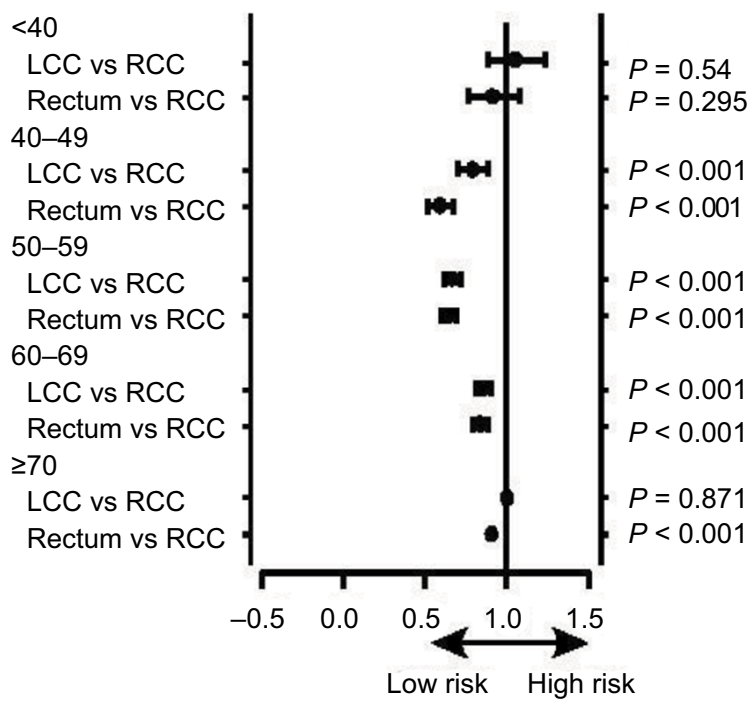

Figure 3 (A-C) The survival difference of RCC, LCC, and rectosigmoid colon cancer in the African-American, White, and Asian/Pacific Islander patients. Abbreviations: LCC, left-sided colon cancer; RCC, right-sided colon cancer.

Additionally, trend analysis of incidence data between 2004 and 2014 revealed that RCC incidence increased by $23.5 \%$, and the trend was most apparent in White patients, where RCC increased by two to three times in patients aged $\geq 70$ years. That is, right-sided lesions predominate in older age groups, whereas there are more LCC/rectal cancer cases in younger and middle-aged patients, especially among Whites.

Racial disparities in CRC incidence have been established in the literature. ${ }^{4}$ Socioeconomic status, comorbidity rates, and cancer biology could contribute to this. ${ }^{20,21}$ The reason for the ethnicity difference in terms of tumor location in younger patients is unclear. However, it may have clinical implications and may direct research in the future. Our results support the premise that screening endoscopy has a differential preventive effect on RCC and LCC. Our findings are also supported by Baxter et al, ${ }^{22}$ who reported that colonoscopy reduces mortality in LCC significantly (by $70 \%$ ), but apparently does not have a significant effect on mortality in RCC. External factors, such as overlooked lesions of the proximal colon and/or inadequate coverage 
of the whole colon during colonoscopy, may be pertinent. Fuccio et al reported faster growth of right-sided adenomas as compared to left-sided adenomas, implying a smaller chance for detecting proximal colonic polyps by screening. ${ }^{23}$ However, Meza et al reported the opposite: slower growth of proximal colonic polyps than that of distal colonic and rectal polyps, which is sex specific and consistent with the delayed onset of RCC as opposed to LCC. Our study argues that the biological characteristic differences are predominant in older patients, especially among White patients. For screening CRC in the general population, our findings indicate the importance of screening schedules that distinguish the variances between ethnicities ${ }^{23,24}$ and the possible benefits of differential targeting of screening strategies for right- and left-sided lesions at different ages. Considering these findings, future trials involving colonoscopy should power recruitment appropriately to enable a priori stratified analyses for right- and left-sided neoplastic endpoints. Such modeling improvements will certainly yield more reliable risk predictions, better informing policymakers on the economic strategies for screening and interventions for preventing CRC more effectively at a population level.

Wong et al reported that among White patients who received bevacizumab, patients with RCC had the most obvious progression-free survival benefit. ${ }^{10}$ However, Brenner et al revealed the apparent survival advantage in LCC when chemotherapy was combined with bevacizumab. ${ }^{25}$ Moreover, in metastatic CRC in Chinese and wide $K R A S$-wide patients, the combination of cetuximab and chemotherapy enhanced the objective response rate, progression-free survival, and overall survival in LCC cases without survival benefit in RCC. ${ }^{11,12}$ This indicates that patient inclusion in clinical trials should consider not only tumor location, but also ethnicity, given the different reactions of patients of different ethnicities to targeted agents.

Two distinct mechanistic pathways have been suggested in the carcinogenesis of sporadic CRC: chromosomal instability (CIN) and microsatellite instability (MSI) ${ }^{26} \mathrm{CIN}$ is more common in cancers arising from the distal colon and rectum than in those from the proximal colon. ${ }^{26}$ Most sporadic high MSI CRC tends to occur in the proximal (right-side) colon in the elderly. ${ }^{26,27}$ In contrast, sporadic CRC in elderly patients, which have MSI, is usually generated by hypermethylation of a DNA mismatch repair gene, such as $M L H 1$ and the subsequent loss of the MLH1 protein. ${ }^{28}$ Poorly differentiated adenocarcinoma is often observed in high MSI CRC. As this feature of CRC in older White patients resembles that of CRC with MSI in the present study, MSI may be an important carcinogenic pathway of CRC in elderly White patients, and this will be an important matter of future investigation. Therefore, we may speculate that the increased prevalence of concomitant adenomas with increased age in the elderly may be associated with colorectal carcinogenesis through the CIN pathway.

In the African-American patients, there was no survival difference between RCC and LCC among all age groups. Similarly, in Asian/Pacific Islander patients, RCC survival was poorest only in the 50-59 years age group. However, in White patients, RCC survival was poorest in the age groups $40-49,50-59,60-69$, and $\geq 70$ years; the tumor location survival difference was more apparent only in White patients. The potential reason remains unknown, although income difference and socioeconomic status are the possible reasons. White patients present with later-stage cancer and are more likely to undergo cancer-directed surgery than patients of other ethnicities, and LCC itself could reflect a survival advantage. ${ }^{29}$

There are some limitations to our study. First, its retrospective nature renders the data potentially vulnerable to bias. We were unable to properly assess personal and family history of CRC, and we did not evaluate familial adenomatous polyposis. However, one of our exclusion criteria was personal or family history of colorectal adenoma or carcinoma, although previous reports did not stipulate this exclusion criterion. ${ }^{30,31}$ Hence, our cohort may sufficiently represent the average risk population with reasonably generalizable prevalence estimates. The other limitation is that socioeconomic status is not available in the SEER database, but socioeconomic differences exist in different races and they may influence the survival and diagnosis of CRC.

Lastly, there were differences between the younger and older patient populations, with adverse factors being more common in patients aged $<70$ years. Moreover, the frequency of RCC differed according to age and ethnicity, wherein the frequency of RCC was relatively high in all ethnicities studied, especially in White patients.

\section{Conclusion}

The study found more adverse prognosis in younger patients compared to older patients. Tumor location frequency differed based on age; the most pronounced differences were found in White patients. There was relatively high RCC incidence in patients from all ethnicities studied, especially in White patients. The RCC survival inferiority was present only in White patients. 


\section{Data sharing statement}

All data were retrieved from the Surveillance, Epidemiology, and End Results (SEER) program of the National Cancer Institute between 2004 and 2012. The datasets are available in the SEER dataset repository https://seer.cancer.gov/ and the patients' data were anonymized.

\section{Acknowledgment}

This work was supported by the Natural Science Foundation of Guangdong, China (grant number 2015A030313010).

\section{Authors contributions}

YL, XZC, GGF, and XLP made substantial contributions to study conception and design; YL and XZC collected the data; YL, XZC, GGF, and XLP analyzed the data and drafted the manuscript; HWZ, KPF, XQK, and JC analyzed the data; YL granted final approval of the version to be published; XXJ and XLP revised the manuscript critically for important intellectual content; and XLP agreed to be accountable for all aspects of the work and for ensuring questions related to the accuracy or integrity of this work were appropriately investigated and resolved. All authors contributed toward data analysis, drafting and revising the paper and agree to be accountable for all aspects of the work.

\section{Disclosure}

The authors report no conflicts of interest in this work.

\section{References}

1. Ferlay J, Shin HR, Bray F, Forman D, Mathers C, Parkin DM. Estimates of worldwide burden of cancer in 2008: GLOBOCAN 2008. Int $J$ Cancer. 2010;127(12):2893-2917.

2. Li FY, Lai MD. Colorectal cancer, one entity or three. J Zhejiang Univ Sci B. 2009;10(3):219-229.

3. Tawk R, Abner A, Ashford A, Brown CP. Differences in Colorectal Cancer Outcomes by Race and Insurance. Int $J$ Environ Res Public Health. 2015;13(1):ijerph13010048.

4. Siegel R, Desantis C, Jemal A. Colorectal cancer statistics, 2014. CA Cancer J Clin. 2014;64(2):104-117.

5. Alexander DD, Waterbor J, Hughes T, Funkhouser E, Grizzle W, Manne U. African-American and Caucasian disparities in colorectal cancer mortality and survival by data source: an epidemiologic review. Cancer Biomark. 2007;3(6):301-313.

6. Gill AA, Zahm SH, Shriver CD, Stojadinovic A, Mcglynn KA, Zhu K. Colon cancer lymph node evaluation among military health system beneficiaries: an analysis by race/ethnicity. Ann Surg Oncol. 2015;22(1):195-202.

7. Siegel RL, Miller KD, Fedewa SA, et al. Colorectal cancer statistics, 2017. CA Cancer J Clin. 2017;67(3):177-193.

8. Ito Y, Nakayama T, Miyashiro I, et al. Trends in 'cure' fraction from colorectal cancer by age and tumour stage between 1975 and 2000, using population-based data, Osaka, Japan. Jpn J Clin Oncol. 2012;42(10):974-983.

9. Shah AB, Sarfati D, Blakely T, Atkinson J, Dennett ER. Trends in colorectal cancer incidence rates in New Zealand, 1981-2004. ANZ J Surg. 2012;82(4):258-264.
10. Wong HL, Lee B, Field K, et al. Impact of Primary Tumor Site on Bevacizumab Efficacy in Metastatic Colorectal Cancer. Clin Colorectal Cancer. 2016;15(2):e9-e15.

11. Wang F, Bai L, Liu TS, et al. Right-sided colon cancer and left-sided colorectal cancers respond differently to cetuximab. Chin J Cancer. 2015;34(9):384-393.

12. Brulé SY, Jonker DJ, Karapetis CS, et al. Location of colon cancer (right-sided versus left-sided) as a prognostic factor and a predictor of benefit from cetuximab in NCIC CO.17. Eur J Cancer. 2015;51(11):1405-1414.

13. Nahleh ZA. Hormonal therapy for male breast cancer: A different approach for a different disease. Cancer Treat Rev. 2006;32(2):101-105.

14. Liang PS, Mayer JD, Wakefield J, Ko CW, Cw K. Temporal Trends in Geographic and Sociodemographic Disparities in Colorectal Cancer Among Medicare Patients, 1973-2010. J Rural Health. 2017;33(4):361-370.

15. Siegel R, Naishadham D, Jemal A. Cancer statistics, 2013. CA Cancer J Clin. 2013;63(1):11-30.

16. Siegel R, Naishadham D, Jemal A. Cancer statistics, 2014. CA Cancer J Clin. 2014;64(1):9-29.

17. Fairley TL, Cardinez CJ, Martin J, et al. Colorectal cancer in U.S. adults younger than 50 years of age, 1998-2001. Cancer. 2006;107(5 Suppl):1153-1161

18. Mostafa G, Matthews BD, Norton HJ, Kercher KW, Sing RF, Heniford BT. Influence of demographics on colorectal cancer. Am Surg. 2004;70(3):259-264.

19. O'Connell JB, Maggard MA, Liu JH, Etzioni DA, Livingston EH, Ko $\mathrm{CY}, \mathrm{Cy} \mathrm{K}$. Do young colon cancer patients have worse outcomes? World J Surg. 2004;28(6):558-562.

20. Eloranta S, Lambert PC, Cavalli-Bjorkman N, Andersson TM, Glimelius B, Dickman PW. Does socioeconomic status influence the prospect of cure from colon cancer--a population-based study in Sweden 1965-2000. Eur J Cancer. 2010;46(16):2965-2972.

21. Gunnarsson H, Ekholm A, Olsson LI. Emergency presentation and socioeconomic status in colon cancer. Eur J Surg Oncol. 2013;39(8):831-836.

22. Baxter NN, Goldwasser MA, Paszat LF, Saskin R, Urbach DR, Rabeneck L. Association of colonoscopy and death from colorectal cancer. Ann Intern Med. 2009;150(1):1-8.

23. Fuccio L, Spada C, Frazzoni L, et al. Higher adenoma recurrence rate after left- versus right-sided colectomy for colon cancer. Gastrointest Endosc. 2015;82(2):337-343.

24. Meza R, Jeon J, Renehan AG, Luebeck EG. Colorectal cancer incidence trends in the United States and United Kingdom: evidence of right- to left-sided biological gradients with implications for screening. Cancer Res. 2010;70(13):5419-5429.

25. Brenner H, Hoffmeister M, Stegmaier C, Brenner G, Altenhofen L, Haug U. Risk of progression of advanced adenomas to colorectal cancer by age and sex: estimates based on 840,149 screening colonoscopies. Gut. 2007;56(11):1585-1589.

26. Nojadeh JN, Behrouz Sharif S, Sakhinia E. Microsatellite instability in colorectal cancer. Excli J. 2018;17:159-168.

27. Barresi V, Castorina S, Musso N, et al. Chromosomal instability analysis and regional tumor heterogeneity in colon cancer. Cancer Genet. 2017;210:9-21.

28. Boland CR, Goel A. Microsatellite instability in colorectal cancer. Gastroenterology. 2010;138(6):2073-2087.

29. Armenia SJ, Pentakota SR, Merchant AM. Socioeconomic factors and mortality in emergency general surgery: trends over a 20 -year period. J Surg Res. 2017;212:178-186.

30. Wilschut JA, Habbema JD, Ramsey SD, Boer R, Looman CW, van Ballegooijen $\mathrm{M}$. Increased risk of adenomas in individuals with a family history of colorectal cancer: results of a meta-analysis. Cancer Causes Control. 2010;21(12):2287-2293.

31. del Vecchio Blanco G, Cretella M, Paoluzi OA, et al. Adenoma, advanced adenoma and colorectal cancer prevalence in asymptomatic 40- to 49-year-old subjects with a first-degree family history of colorectal cancer. Colorectal Dis. 2013;15(9):1093-1099. 


\section{Supplementary material}

A

According to stage

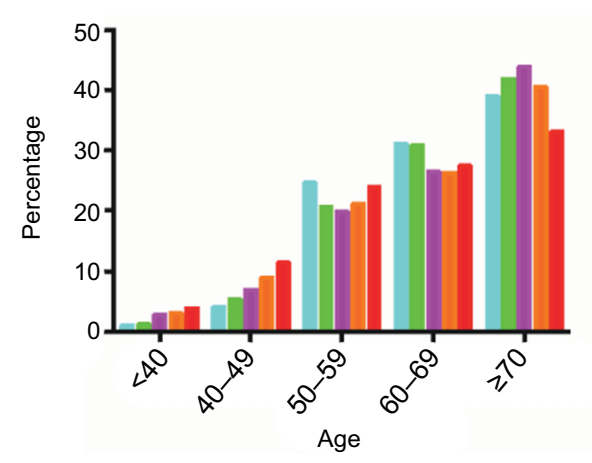

C

According to TNLN

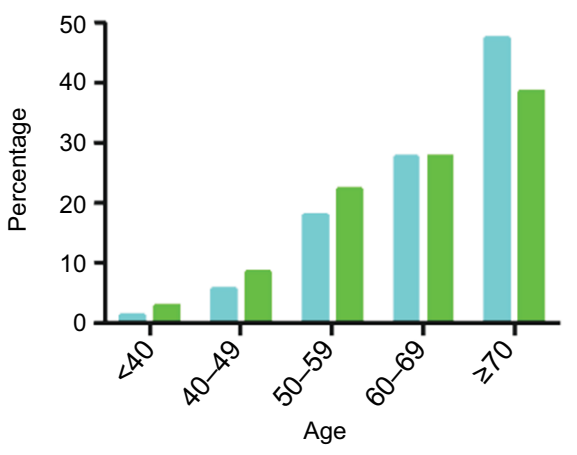

$\mathbf{E}$

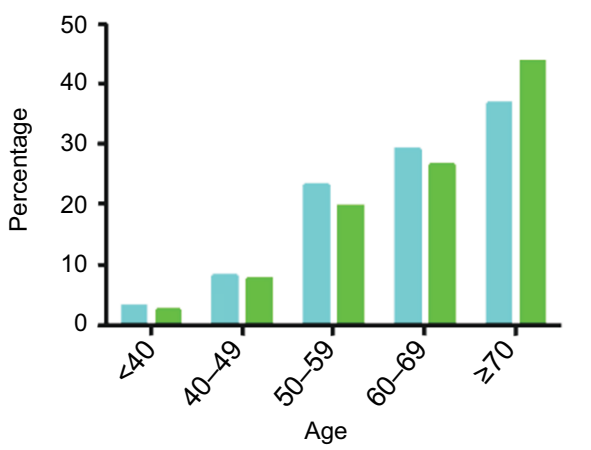

B

According to pathology

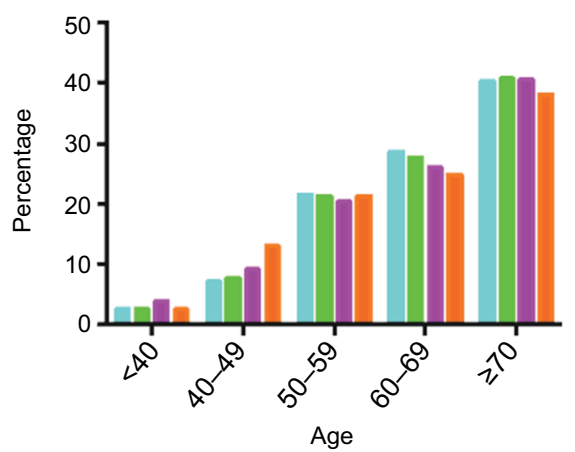

D
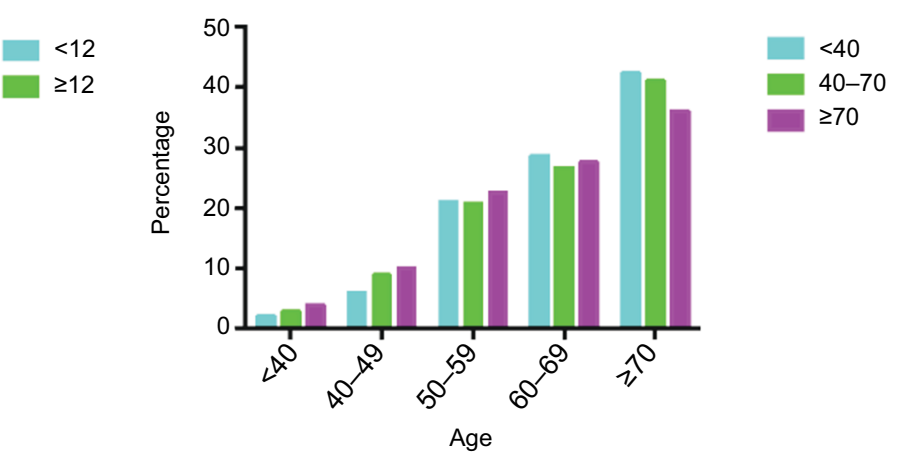

Figure SI Distribution of tumor location according to age group in the African-American subgroups.

Notes: (A) Stage; (B) pathology (differentiation); (C) TNLN; (D) tumor size ( $<40,40-70$, and $\geq 70 \mathrm{~mm}$ ); (E) sex. The shift for the proximal predominance of CRC increased with age.

Abbreviations: CRC, colorectal cancer; TNLN, total number of lymph nodes. 
A

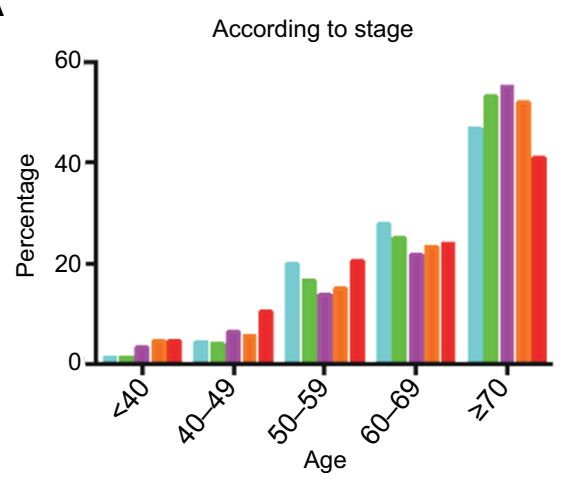

C

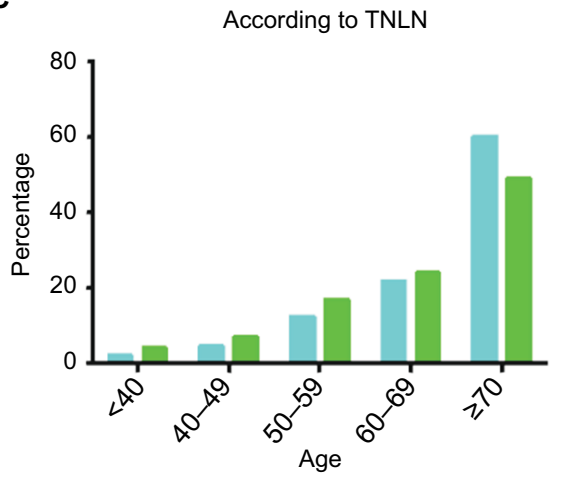

E

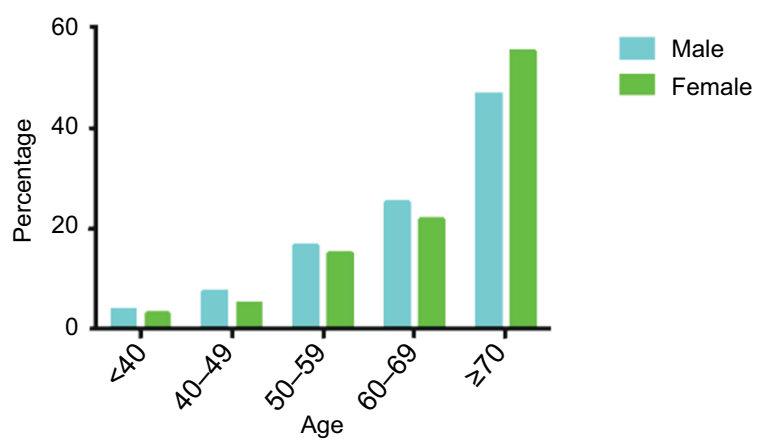

B

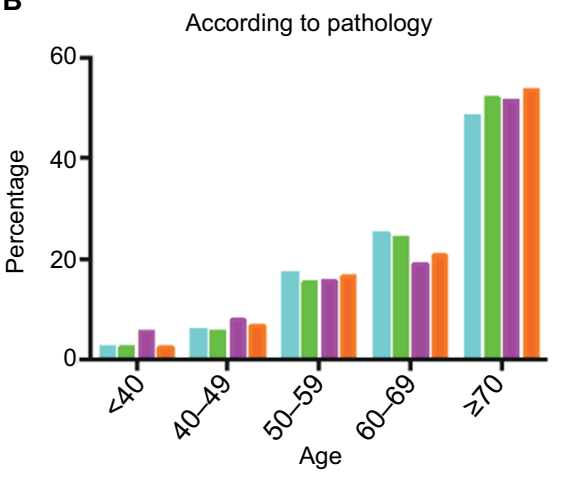

D

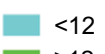

$\geq 12$

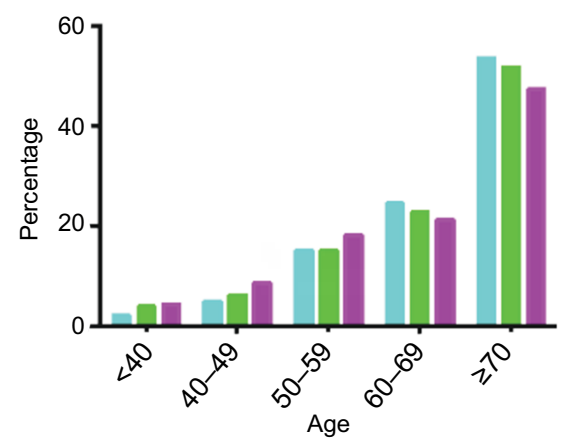

Well

Moderately

Poorly

Undifferentiated

Figure S2 Distribution of tumor location according to age group in the Asian/Pacific Islander subgroups.

Notes: (A) Stage; (B) pathology (differentiation); (C) TNLN; (D) tumor size ( $<40,40-70$, and $\geq 70 \mathrm{~mm}$ ); (E) sex. The shift for the proximal predominance of CRC increased with age.

Abbreviations: CRC, colorectal cancer; TNLN, total number of lymph nodes. 
A

According to stage

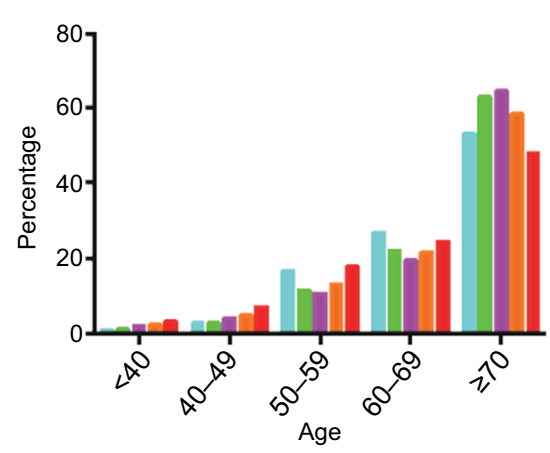

C

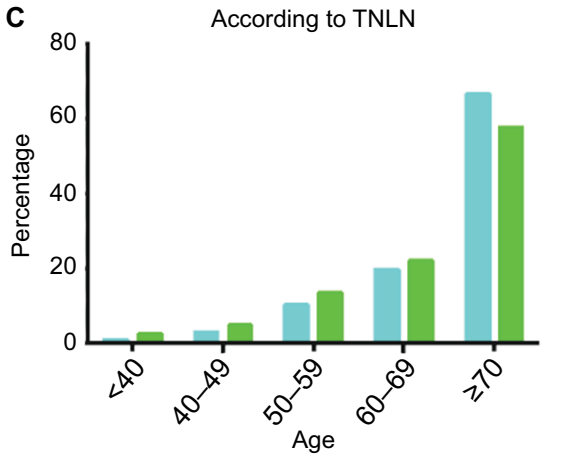

E

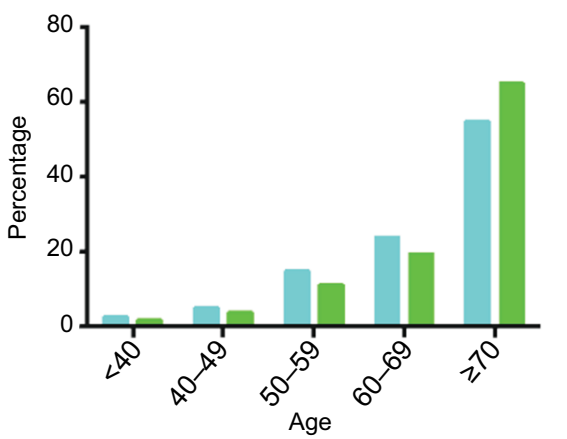

B

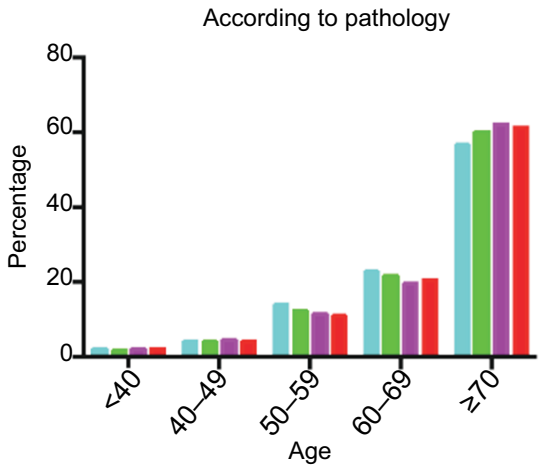

D

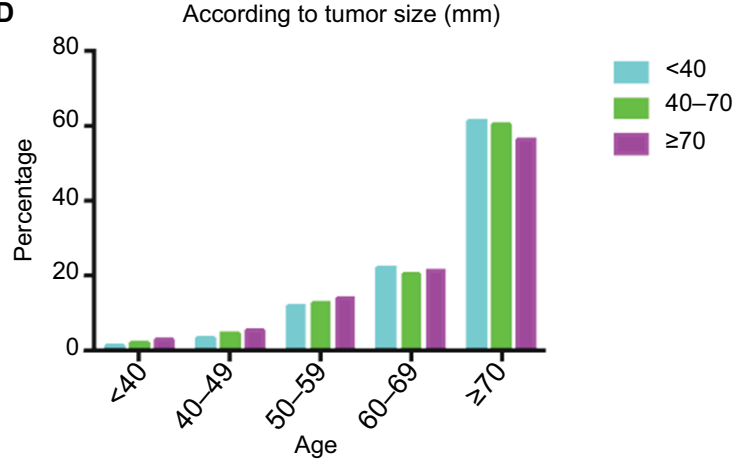

Male

Female

Figure S3 Distribution rate of tumor location according to age group in the White subgroups.

Notes: (A) Stage; (B) pathology (differentiation); (C) TNLN; (D) tumor size (<40, 40-70, and $\geq 70 \mathrm{~mm}$ ); (E) sex. The shift for the proximal predominance of CRC increased with age.

Abbreviations: CRC, colorectal cancer; TNLN, total number of lymph nodes.

Cancer Management and Research

\section{Publish your work in this journal}

Cancer Management and Research is an international, peer-reviewed open access journal focusing on cancer research and the optimal use of preventative and integrated treatment interventions to achieve improved outcomes, enhanced survival and quality of life for the cancer patient. The manuscript management system is completely online and includes

\section{Dovepress}

a very quick and fair peer-review system, which is all easy to use. Visit http://www.dovepress.com/testimonials.php to read real quotes from published authors.

Submit your manuscript here: https://www.dovepress.com/cancer-management-and-research-journal 